\title{
BMJ Open Risk factors for severe outcomes in people with diabetes hospitalised for COVID-19: a cross-sectional database study
}

\author{
Emilio Ortega, ${ }^{1,2,3}$ Rosa Corcoy, ${ }^{4,5,6}$ Mònica Gratacòs, ${ }^{1}$ \\ Francesc Xavier Cos Claramunt, ${ }^{1,7}$ Manel Mata-Cases (D) ,1,8,9 \\ Ramon Puig-Treserra, ${ }^{1}$ Jordi Real, ${ }^{1}$ Bogdan Vlacho, ${ }^{1}$ Esmeralda Castelblanco (D) , ${ }^{1,9}$ \\ Pere Domingo, ${ }^{10}$ Kamlesh Khunti, ${ }^{11}$ Josep Franch-Nadal, ${ }^{1,9,12}$ \\ Didac Mauricio (D) 1,4,9,13
}

To cite: Ortega E, Corcoy R, Gratacòs $\mathrm{M}$, et al. Risk factors for severe outcomes in people with diabetes hospitalised for COVID-19: a cross-sectional database study. BMJ Open 2021;11:e051237. doi:10.1136/ bmjopen-2021-051237

- Prepublication history and additional supplemental material for this paper are available online. To view these files, please visit the journal online (http://dx.doi.org/10.1136/ bmjopen-2021-051237)

Received 15 March 2021 Accepted 01 July 2021

Check for updates

(C) Author(s) (or their employer(s)) 2021. Re-use permitted under CC BY-NC. No commercial re-use. See rights and permissions. Published by BMJ.

For numbered affiliations see end of article.

Correspondence to Dr Didac Mauricio; didacmauricio@gmail.com and Dr Josep Franch-Nadal; josep.franch@gmail.com

\section{ABSTRACT}

Aim This study's objective was to assess the risk of severe in-hospital complications of patients admitted for COVID-19 and diabetes mellitus (DM).

Design This was a cross-sectional study.

Settings We used pseudonymised medical record data provided by six general hospitals from the HM Hospitales group in Spain.

Outcome measures Multiple logistic regression analyses were used to identify variables associated with mortality and the composite of mortality or invasive mechanical ventilation (IMV) in the overall population, and stratified for the presence or absence of DM. Spline analysis was conducted on the entire population to investigate the relationship between glucose levels at admission and outcomes.

Results Overall, 1621 individuals without DM and 448 with DM were identified in the database. Patients with DM were on average 5.1 years older than those without. The overall in-hospital mortality was $18.6 \%(\mathrm{~N}=301)$, and was higher among patients with DM than those without $(26.3 \%$ vs $11.3 \% ; p<0.001)$. DM was independently associated with death, and death or IMV $(\mathrm{OR}=2.33,95 \% \mathrm{Cl}: 1.7$ to 3.1 and $\mathrm{OR}=2.11,95 \% \mathrm{Cl}: 1.6$ to 2.8 , respectively; $\mathrm{p}<0.001$ ). In subjects with DM, the only variables independently associated with both outcomes were age $>65$ years, male sex and pre-existing chronic kidney disease. We observed a non-linear relationship between blood glucose levels at admission and risk of in-hospital mortality and death or IMV. The highest probability for each outcome (around $50 \%$ ) was at random glucose of around $550 \mathrm{mg} / \mathrm{dL}$ (30.6 $\mathrm{mmol} / \mathrm{L}$ ), and the risks flattened above this value.

Conclusion The results confirm the high burden associated with DM in patients hospitalised with COVID-19 infection, particularly among men, the elderly and those with impaired kidney function. Moreover, hyperglycaemia on admission was strongly associated with poor outcomes, suggesting that personalised optimisation could help to improve outcome during the hospital stay.

\section{INTRODUCTION}

On 30 January 2020, the WHO declared the outbreak of the novel SARS-CoV-2 coronavirus

\section{Strengths and limitations of this study}

- A major strength of our study is the thorough methodological approach to analyse the risk of inhospital COVID-19-related complications based on the presence of diabetes mellitus (DM) or overt hyperglycaemia.

- We were limited by not having access to the patients' medical history prior to admission, and the low number of registers for some important DM variables (such as glycosylated haemoglobin ( $\mathrm{Hb} 1 \mathrm{Ac})$ ), and the lack of data on weight or body mass index (only the presence of obesity).

- The selection of subjects with DM was made based on a proxy algorithm including DM diagnosis during the hospital stay, antidiabetic treatment, and $\mathrm{HbA1C}$ and blood glucose levels at admission.

- We used random blood glucose on admission for spline analyses, thus preventing the distinction between stress-related hyperglycaemia and uncontrolled pre-existing DM.

pandemic, a public health emergency of international importance. A few days later, the respiratory disease caused by SARS-CoV-2 was officially named COVID-19. ${ }^{2}$ The first person diagnosed as positive in Spain was confirmed on 31 January 2020, on the island of La Gomera. ${ }^{3}$ The median age of hospitalised patients infected with SARS-CoV-2 is 46.2 years, men comprise about $60 \%$ of patients, and the average incubation period is 5.7 days. ${ }^{4}$ As of 8 February 2021, approximately 3 million people have been infected with SARS-CoV-2 in Spain since the start of the COVID-19 pandemic, and 62295 persons have died.

Several meta-analyses have reported that the most severe and fatal cases of COVID-19 
occur among the elderly and in patients with underlying comorbidities. ${ }^{5-7}$ Indeed, those with two or more concomitant diseases have a significantly higher risk of admission to an intensive care unit (ICU), invasive ventilation, or death compared with those with a single concomitant disease, or without comorbidities. ${ }^{8}$ The most prevalent comorbidities associated with increased COVID-19-related morbidity and mortality are the presence of diabetes mellitus (DM), cardiovascular diseases (CVDs), chronic lung disease, chronic kidney disease (CKD), hypertension, cancer and obesity. ${ }^{5-7}$ In addition, the $\mathrm{AB} 0$ blood type may play a role in the susceptibility and severity of COVID-19 infection, which could be of importance in patients with underlying high-risk conditions. ${ }^{8}$ For instance, it has been reported that non-0 blood group patients with hypertension have significantly higher values of pro-thrombotic indexes and increased rates of cardiac injury and deaths compared with 0 patients. ${ }^{9}$

SARS-CoV-2 uses ACE-2 as a cellular entry receptor, and the spike protein of the virus needs to be cleaved by cellular proteases (specifically TMPRSS2) to fuse with the cellular membrane. ${ }^{10}$ Although it was initially assumed that ACE inhibitors and angiotensin receptor blockers to treat hypertension or cardiovascular conditions might exacerbate COVID-19 infection and lead to worse outcomes, the most recent available meta-analysis did not confirm this higher risk. ${ }^{11}$ Finally, it has been suggested that modulating TMPRSS2 expression through specific antibodies or non-coding RNAs could prevent virus entry into host cells, ${ }^{11}{ }^{12}$ but these potential therapeutic options are still under investigation.

Previous studies have reported that people with DM are prone to new infections and recurrence, particularly influenza and pneumonia, due to impaired defences and disease complications. ${ }^{13-16}$ Although the estimated prevalence of DM in COVID-19-infected patients varies greatly by geographical region, it is considered similar to the DM prevalence in the general population, thus not representing a risk factor for infection. ${ }^{17}$ However, the prevalence of diabetes among COVID-19 hospitalised subjects is higher than the overall diabetes prevalence. ${ }^{17}{ }^{18} \mathrm{~A}$ study conducted in England found that one-third of in-hospital deaths occurred in people with type $2 \mathrm{DM}$ and that these patients had greater odds of COVID-19-related in-hospital death than those without DM. ${ }^{19}$ This observation has been confirmed in a meta-analysis showing that DM is associated with a twofold higher risk of dying from COVID- $19,{ }^{20}$ and a second study reporting that patients with pre-existing DM have a threefold greater risk of in-hospital mortality. ${ }^{21}$

Early reports showed that about half of patients with severe COVID-19 presented acute hyperglycaemia, with no more than $10 \%$ of them having a prior diagnosis of DM. ${ }^{22}{ }^{23}$ Following these observations, two meta-analyses concluded that hyperglycaemia at hospital admission is associated with severe complications and mortality, regardless of diabetes status. ${ }^{24} 25$ Moreover, hyperglycaemia also has a negative impact on the therapeutic response to tocilizumab in patients with COVID-19-related systemic inflammation. ${ }^{26}$

In Spain, DM is a highly prevalent disease in people over 18 years of age (13.8\% of the population) ${ }^{27}$ Given the high prevalence of DM and the additional challenging scenario that COVID-19 poses to healthcare professionals in this particular population, it is crucial to accumulate and share information and data from different countries and regions. ${ }^{28}$ Therefore, the main objective of this study was to assess the risk of in-hospital COVID-19-related complications based on the presence of DM or overt hyperglycaemia at admission in Spain.

\section{METHODS}

\section{Study design and settings}

This was a cross-sectional study in hospitalised individuals infected with SARS-CoV-2, stratified by presence or absence of DM. Data were obtained from pseudonymised electronic health records provided by six general hospitals from the HM Hospitales group (Spain). The database included information related during the hospital stay (diagnosis and procedure codes, prescribed medications, vital signs and laboratory parameters), from 2310 subjects during the first COVID-19 wave with hospital admission between 27 January and 24 April 2020 (study start and end date, respectively). Subjects were followed from admission to hospital discharge or death. Detailed information related to the database is presented in the online supplemental material (database description). The REporting of studies Conducted using Observational Routinely-collected Data Checklist is presented as online supplemental material.

The study data were collected by medical professionals of the HM Hospitales group (Spain) during the first wave of the COVID-19 pandemic. In order to promote COVID-19-related research, the HM Hospitales group pseudonymised the medical history of SARS-CoV-2infected patients and created a project titled: 'Covid Data Save Lives'. Before access was granted, a formal petition, specific study protocol and ethics committee approval were obtained.

\section{Inclusion and exclusion criteria}

The study enrolled people over 18 years of age with SARS-CoV-2 infection (COVID-19 positive) microbiologically proven by reverse transcription PCR (RT-PCR). Those with DM were identified in the database if they: (1) had any ICD-10 (International Statistical Classification of Diseases) diagnostic code for type 1 or type $2 \mathrm{DM}$ (ie, E.10 and E.11), (2) were on treatment with antidiabetic drugs, (3) had a register of insulin use within the first 24 hours after admission, or (4) had a glycosylated haemoglobin (HbAlc) value $\geq 6.5 \%$ ( $48 \mathrm{mmol} / \mathrm{mol}$; first available record after admission) or baseline blood glucose (BG) values $\geq 200 \mathrm{mg} / \mathrm{dL} \quad(11.1 \mathrm{mmol} / \mathrm{L}$; recorded within the first 24 hours of admission). Subjects with no 
confirmation of SARS-CoV-2 infection and those younger than 18 years were excluded from the study.

\section{Study variables}

The following baseline variables were collected: age and sex; SARS-CoV-2 diagnosis (positive RT-PCR); comorbidities (ie, hypertension, hyperlipidaemia, obesity (body mass index (BMI) $\left.\geq 30 \mathrm{~kg} / \mathrm{m}^{2}\right)$, CVD, heart failure, cerebrovascular diseases, ischaemic heart disease, CKD, chronic obstructive pulmonary disease (COPD), asthma, mental disorders and cancer); blood laboratory parameters (ie, HbA1c, BG, electrolytes, renal function, liver function, haematology and coagulation, inflammation markers and gas tests); clinical parameters (ie, systolic and diastolic blood pressure, heart rate and temperature); and concomitant medications (ie, baseline insulins, systemic corticosteroids, antimicrobials, anticoagulants and antiplatelet agents, and antihypertensive and lipidlowering drugs).

We considered the following variables as events or complications during the hospital stay: death, acute respiratory distress syndrome (ARDS), pulmonary thrombosis, neurological complications (including encephalopathy, encephalitis, myelitis and encephalomyelitis), thrombotic complications identified by ICD-10 diagnostic codes (phlebitis and thrombophlebitis), admission to ICU and invasive mechanical ventilation (IMV) identified by ICD-10 procedure codes. The composite primary outcome was defined as death or IMV.

\section{Statistical methods}

The demographic and clinical characteristics of the two groups of hospitalised patients (ie, with or without DM) were compared and summarised at the quantitative (minimum, maximum, median, first and third quartile, mean and SD) or categorical level (frequency, number and $\%)$.

The association between the study outcomes (ie, mortality and mortality or mechanical ventilation) and DM was performed using logistic regression analyses adjusted for sex, age and associated risk factors. In addition, several models of interest were tested (a model with basic clinical variables such as age and sex, a model adding obesity, hypertension and hyperlipidaemia, and a model adding organ lesion variables, such as CVD, heart failure, CKD, COPD), namely with the sequential inclusion of different covariates and the estimated differences expressed as OR and the respective 95\% CIs. We evaluated goodness of fit of the logistic regression models with Hosmer-Lemeshow test. To analyse the non-linear relationship of random $\mathrm{BG}$ levels on admission with the two study outcomes, we used an adjusted semiparametric model (generalised additive model) calculating the spline curves with two df (knots) using the mgcv package in R, V.1.8-31 ${ }^{29}$ with adjustment for potential confounders. We analysed the entire database available and no statistical power was calculated. Data management and statistical analyses were performed using the $\mathrm{R}$ statistical software V.3.6.1 (https://www.r-project.org/).

\section{Patient and public involvement}

Patients or the public were not involved in the design, or conduct, or reporting, or dissemination plans of our research.

\section{RESULTS}

\section{Baseline characteristics}

Of the 2306 subjects admitted to hospital within the period of study, 2069 were over 18 years of age and had a positive diagnostic test for SARS-CoV-2 (figure 1). Among them, $448(21.7 \%)$ were identified as having DM and 1621 (78.3\%) without DM (non-DM group). The characteristics of the two populations at hospital admission are shown in table 1 . Subjects with DM were on average 5.1 years older than subjects without DM, and more frequently men ( $67.9 \%$ vs $58.6 \%$ ). Moreover, individuals in the DM group had a poor comorbidity profile, with a higher frequency of all assessed prior conditions except for cerebrovascular diseases and asthma.

Regarding laboratory parameters on admission (online supplemental table 1), the DM group had slightly lower estimated glomerular filtration rates (eGFRs) $\left(73.5 \pm 26.5 \mathrm{~mL} / \mathrm{min} / 1.73 \mathrm{~m}^{2}\right.$ vs $81.2 \pm 23.9 \mathrm{~mL} / \mathrm{min} / 1.73$ $\left.\mathrm{m}^{2} ; \mathrm{p}<0.001\right)$, and higher levels of serum creatinine $(1.09 \pm 0.72 \mathrm{mg} / \mathrm{dL}$ vs $0.94 \pm 0.51 \mathrm{mg} / \mathrm{dL} ; \mathrm{p}<0.001)$ than the non-DM group. Regarding markers of inflammation and infection, we observed higher levels of $\mathrm{C}$ reactive protein and procalcitonin in the DM group $(97.1 \pm 107 \mathrm{mg} / \mathrm{L}$ vs $75.9 \pm 82.5 \mathrm{mg} / \mathrm{L}$ and $0.66 \pm 1.30 \mathrm{mg} / \mathrm{L}$ vs $0.39 \pm 1.30$ $\mathrm{mg} / \mathrm{L}$, respectively; $\mathrm{p}<0.001$ ). We also observed higher levels of D-dimer, a marker of endothelial and coagulation dysfunction, in the DM group (3990 $\pm 10800 \mathrm{ng} /$ $\mathrm{mL}$ vs $2340 \pm 6720 \mathrm{ng} / \mathrm{mL}$, respectively). Regarding the pharmacological therapy used during the hospital stay, we observed differences and increased use of almost all drugs of interest among subjects with DM, compared with those without DM, especially for diuretics, systemic corticosteroids and tocilizumab.

\section{Events and complications during in-hospital stay}

A total of $301(14.5 \%)$ subjects positive for SARS-CoV-2 died in hospital, 118 (26.3\%) out of 448 in the DM group and $183(11.3 \%)$ out of 1621 in the non-DM group $(p<0.001$; figure 2$)$. All studied events, except pulmonary embolism and thrombotic or neurological complications, were significantly more frequent among patients with DM than those without (figure 2). The most frequent outcome was the composite of death or IMV (31\% in the DM group vs $14 \%$ in the non-DM group; figure 2) followed by death ( $26.3 \%$ vs $11.3 \%)$, admission to ICU (21\% vs $6.9 \%)$, IMV ( $10.7 \%$ vs $4.2 \%)$ and ARDS (3.8\% vs $1.5 \%)$.

The frequency of events by group and age showed that, in both subjects with and without DM, death and the composite of death or IMV were significantly more 


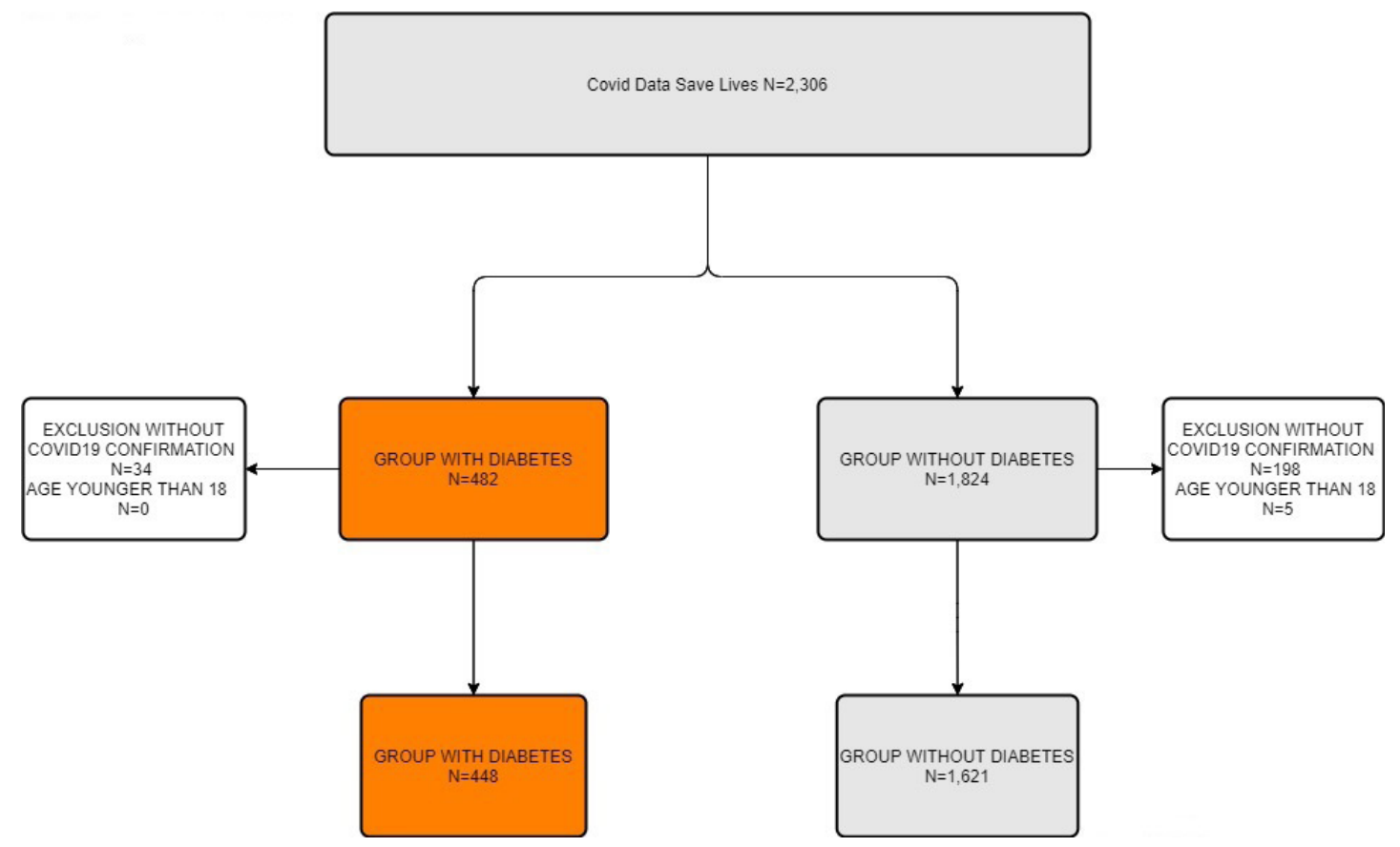

Figure 1 Flow chart diagram.

frequent among those $>65$ years (online supplemental figure 1). In contrast, the proportion of subjects requiring IMV and ICU admission was significantly higher among those $\leq 65$ years and with DM, while age was not significant in those without DM. When stratifying the results by sex, we did not observe differences except for admission to ICU, which was significantly more frequent among male subjects with DM (online supplemental figure 1). Within the diabetes group, when we stratified by pre-existing DM (DM codes and/or HBA1c $\geq 6.5 \%$ and/or antidiabetic treatment) and 'stress' hyperglycaemia/unknown diabetes (glucose $\geq 200 \mathrm{mg} / \mathrm{dL}$ or insulin use within the first 24 hours period after admission), we observed higher percentages for death, death or IMV, ARDS, admission to ICU and IMV events in subjects with 'stress' hyperglycaemia. The results of this stratification are presented in online supplemental table 2.

\section{Baseline demographic and clinical characteristics associated with in-hospital death and death or IMV}

For the overall hospitalised population, the demographic characteristics significantly associated with mortality were male sex and older age $(\mathrm{OR}=1.98,95 \% \mathrm{CI}=1.2$ to 3.3 and $\mathrm{OR}=1.10,95 \% \mathrm{CI}=1.08$ to 1.11 , respectively) (figure 3; online supplemental table 3 ). The comorbidities independently associated with increased odds of death were $\mathrm{DM}(\mathrm{OR}=2.33,95 \% \mathrm{CI}=1.7$ to 3.1$), \mathrm{CKD}(\mathrm{OR}=2.14,95 \%$ $\mathrm{CI}=1.2$ to 3.7$)$ and $\mathrm{COPD}(\mathrm{OR}=1.72,95 \% \mathrm{CI}=1.1$ to 2.8$)$.

When considering the composite outcome of death or IMV, the same variables associated with death (ie, age, sex, diabetes, CKD and COPD) were identified as increasing the risk. In addition, obesity emerged as an independently associated variable $(\mathrm{OR}=1.98,95 \% \mathrm{CI}=1.5$ to 2.7 ) (figure 3, online supplemental table 3).
The multiple logistic regression models were repeated to rule out the potential interaction of DM with different clinical conditions (ie, obesity, hyperlipidaemia, obesity and hyperlipidaemia, heart failure, CKD and COPD) for the in-hospital death outcome. The results showed that none of these conditions affected the relationship between the risk of death and DM (online supplemental table 4).

\section{Factors associated with hospital death and death or IMV by comorbid diabetes}

A subanalysis was performed separately for subjects with or without DM. In the DM group, the only variables independently associated with the risk of both mortality and death or IMV were male sex, older age and CKD (figure 4A and online supplemental tables 5 and 6). In contrast, in subjects without DM, besides the aforementioned variables, the odds of death were also increased among subjects with CVD (OR=1.94, 95\% CI=1.03 to 3.7), and the odds of death or IMV among those with obesity or COPD (OR=2.96, 95\% $\mathrm{CI}=1.7$ to 5.3 and $\mathrm{OR}=2.30$, $95 \% \mathrm{CI}=1.4$ to 3.8 , respectively) (figure $4 \mathrm{~B}$ and online supplemental tables 5 and 6 ).

Factors associated with hospital death and death or IMV by glucose levels at admission

We used non-parametric logistic regression models to assess whether there was a relationship between random BG on admission and the risk of mortality (and death or IMV). We observed a marked non-linearity in the effect of BG on admission in the risk of both outcomes (figure 5A,B and online supplemental table 7). While the risk was increased among subjects with high random BG levels on admission, the magnitudes of the associated mortality differed depending on the baseline 
Table 1 Baseline characteristics of the studied cohorts at hospital admission

\begin{tabular}{|c|c|c|c|c|}
\hline Characteristic & $\begin{array}{l}\text { Overall study } \\
\text { population } \\
\mathrm{N}=2069\end{array}$ & $\begin{array}{l}\text { Diabetes } \\
\mathrm{N}=448\end{array}$ & $\begin{array}{l}\text { No diabetes } \\
\mathrm{N}=1621\end{array}$ & $P$ value \\
\hline Age, mean (SD), years & $67.8(15.7)$ & 71.7 (11.9) & $66.6(16.3)$ & $<0.001$ \\
\hline Age, median (P25, P75), years & $69.0(57.0,80.0)$ & $72.0(64.0,80.0)$ & $67.0(55.0,79.0)$ & $<0.001$ \\
\hline Sex (male), $\mathrm{n}(\%)$ & $1205(60.3)$ & $304(67.9)$ & $950(58.6)$ & $<0.001$ \\
\hline Glucose, mean, (SD) & & & & $<0.001$ \\
\hline $\mathrm{mg} / \mathrm{dL}$ & $124(47.7)$ & $168(74.4)$ & $112(24.8)$ & \\
\hline $\mathrm{mmol} / \mathrm{L}$ & $6.8(2.6)$ & $9.3(4.1)$ & $6.2(1.4)$ & \\
\hline \multicolumn{5}{|l|}{ Comorbidities, n (\%) } \\
\hline Hypertension & $651(31.5)$ & $224(50.0)$ & $427(26.3)$ & $<0.001$ \\
\hline Hyperlipidaemia & $409(19.8)$ & $154(34.4)$ & $255(15.7)$ & $<0.001$ \\
\hline Obesity & $117(5.65)$ & $45(10.0)$ & $72(4.44)$ & $<0.001$ \\
\hline Cardiovascular diseases & 77 (3.72) & $28(6.25)$ & 49 (3.02) & 0.002 \\
\hline Heart failure & $51(2.46)$ & $18(4.02)$ & $33(2.04)$ & 0.026 \\
\hline Cerebrovascular diseases & $27(1.30)$ & $10(2.23)$ & $17(1.05)$ & 0.086 \\
\hline Ischaemic heart disease & $47(2.27)$ & $18(4.02)$ & $29(1.79)$ & 0.009 \\
\hline Chronic kidney disease & $76(3.67)$ & $30(6.70)$ & $46(2.84)$ & $<0.001$ \\
\hline Chronic obstructive pulmonary disease & $112(5.41)$ & 34 (7.59) & $78(4.81)$ & 0.029 \\
\hline Asthma & $2(0.10)$ & $0(0.00)$ & $2(0.12)$ & 1.000 \\
\hline Mental disorders & $114(5.51)$ & $35(7.81)$ & $79(4.87)$ & 0.022 \\
\hline Cancer & $117(5.65)$ & $36(8.04)$ & $81(5.00)$ & 0.019 \\
\hline \multicolumn{5}{|l|}{ Pharmacological therapy, $\mathrm{n}(\%)$} \\
\hline Biguanides & $66(3.19)$ & $66(14.7)$ & $0(0.00)$ & $<0.001$ \\
\hline Sulfonylureas & $1(0.05)$ & $1(0.22)$ & $0(0.00)$ & 0.217 \\
\hline Dipeptidyl peptidase 4 inhibitors & $11(0.53)$ & $11(2.46)$ & $0(0.00)$ & $<0.001$ \\
\hline Fast-acting insulins & $95(4.5)$ & $66(14.7)$ & $29(1.79)$ & $<0.001$ \\
\hline Intermediate-acting insulins & $9(0.43)$ & $7(1.56)$ & $2(0.12)$ & 0.001 \\
\hline Long-acting insulins & $23(1.11)$ & $20(4.46)$ & $3(0.19)$ & $<0.001$ \\
\hline Antibiotics & $1882(91.0)$ & $421(94.0)$ & $1461(90.1)$ & 0.016 \\
\hline Antithrombotics & $1752(84.7)$ & $396(88.4)$ & $1356(83.7)$ & 0.017 \\
\hline Renin-angiotensin system agents & $523(25.3)$ & $153(34.2)$ & $370(22.8)$ & $<0.001$ \\
\hline Beta blocking agents & $316(15.3)$ & $104(23.2)$ & $212(13.1)$ & $<0.001$ \\
\hline Calcium channel blockers & $384(18.6)$ & $118(26.3)$ & $266(16.4)$ & $<0.001$ \\
\hline Diuretics & $508(24.6)$ & $185(41.3)$ & $323(19.9)$ & $<0.001$ \\
\hline Statins & $256(12.4)$ & $88(19.6)$ & $168(10.4)$ & $<0.001$ \\
\hline Systemic corticosteroids & $977(47.2)$ & 267 (59.6) & $710(43.8)$ & $<0.001$ \\
\hline Tocilizumab & $421(20.3)$ & $137(30.6)$ & $284(17.5)$ & $<0.001$ \\
\hline
\end{tabular}

$\mathrm{P} 25, \mathrm{P} 75=25$ th and 75 th percentile, respectively.

values, with a large increase in the log odds of death or IMV with values up to $200 \mathrm{mg} / \mathrm{dL}(11.1 \mathrm{mmol} / \mathrm{L})$, and smaller increases above this level. The logistic regression models (figure 6A,B) showed that the highest probability of death (near $50 \%$ ) was at around $550 \mathrm{mg} /$ $\mathrm{dL}(30.6 \mathrm{mmol} / \mathrm{L})$ and, above this value, the mortality risk flattened. Finally, the multivariate model showed that, beside glucose at admission, male sex, older age,
CKD and COPD were associated with in-hospital death (online supplemental table 7 ). These variables were linked to death or IMV too, but obesity was an additional risk factor (online supplemental table 7).

\section{DISCUSSION}

Data from this cross-sectional study showed that the COVID-19-related in-hospital death rate was higher 


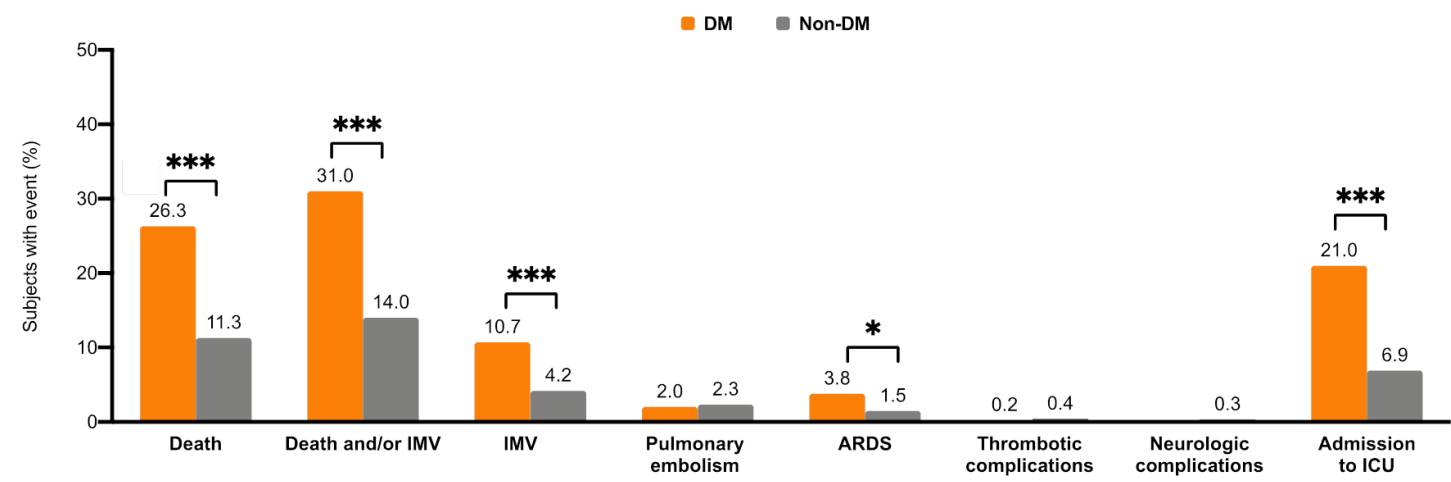

Figure 2 Proportion of events (\%) during hospitalisation according to the presence of diabetes. ${ }^{* * *} \mathrm{P}<0.001 ;{ }^{* *} \mathrm{p}<0.01 ;{ }^{*} \mathrm{p}<0.05$. ARDS, acute respiratory distress syndrome; DM, diabetes mellitus; ICU, intensive care unit; IMV, invasive mechanical ventilation.

among subjects with DM than those without. Moreover, DM was independently associated with the risk of in-hospital case fatality and the composite outcome, death or IMV. In the DM group, both outcomes were associated with older age, male sex and pre-existing CKD. Finally, we observed a non-linear relationship between BG levels on admission and the probability of death and death or IMV in the overall inpatient population.

In our study, the proportion of severe COVID-19 cases (eg, requiring IMV or ICU admission) in the population with DM was higher than in the non-DM cohort.

In-hospital death In-hospital death or IMV

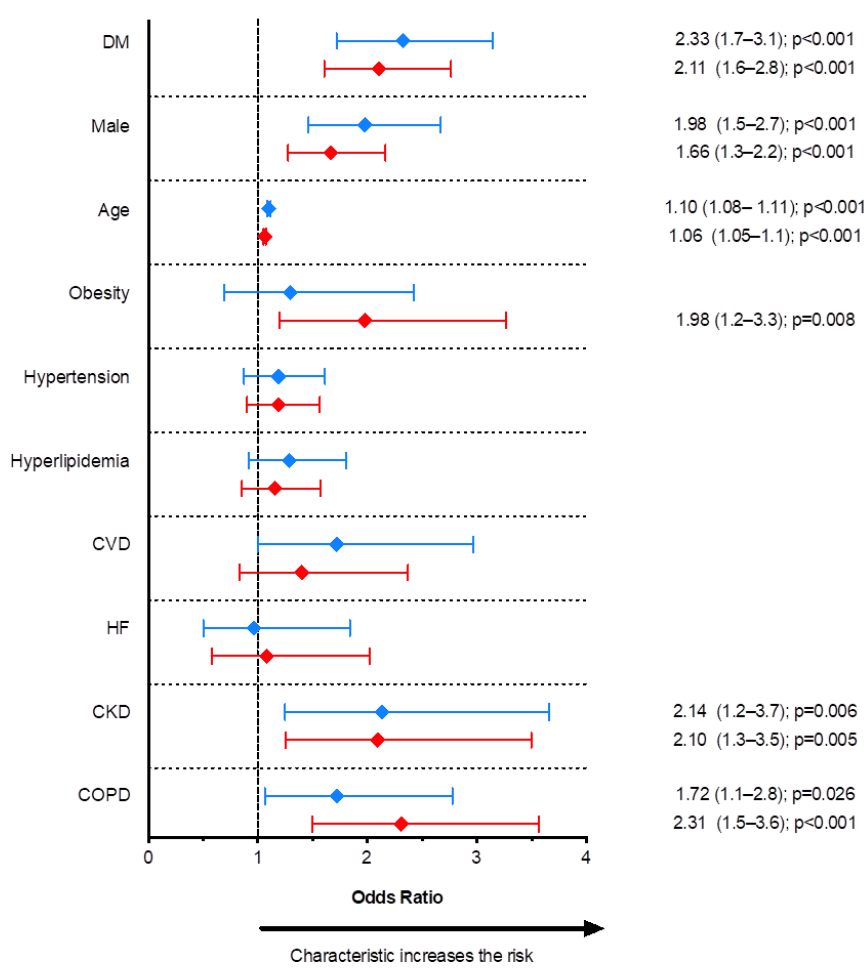

Figure 3 Clinical and demographic variables associated with increased risk of in-hospital death and the composite outcome of death or invasive mechanical ventilation (IMV). CKD, chronic kidney disease; COPD, chronic obstructive pulmonary disease; CVD, cardiovascular disease; DM, diabetes mellitus; HF, heart failure.
Moreover, patients with DM were more frequently men and over 65 years, had more comorbid conditions, and higher levels of inflammatory, endothelial, and coagulation dysfunction markers on admission. Different meta-analyses have reported that older age and male sex are characteristics associated with severe COVID-19 infection and high fatality rates. ${ }^{17} 3031$ Along the same line, studies assessing the phenotypic characteristics of patients with COVID-19 with pre-existing DM have found that those with severe infection were older, had more comorbidities (ie, cerebrovascular disease, CVD, hypertension and COPD), and increased values of inflammation, endothelial and coagulation dysfunction markers (eg, D-dimer, procalcitonin and thrombocytopenia), than those without DM. ${ }^{30-35}$

In our study, patients with DM had significantly higher creatinine on admission, lower eGFR and more frequently pre-existing CKD than subjects without DM. Besides, CKD was the only comorbid condition increasing the odds (threefold increase) of in-hospital death (and death or IMV) among the DM cohort after adjusting for age, sex and confounding variables. Different meta-analyses have identified CKD as a risk factor for severity and in-hospital death in SARS-CoV-2-infected patients. ${ }^{76-38}$ Moreover, a recent study conducted in Danish hospitaldiagnosed patients with COVID-19 reported that kidney insufficiency was independently associated with increased risk of severe disease or death, and the degree of renal impairment inversely correlated with the rate of adverse outcomes. ${ }^{39}$ Although it is difficult to distinguish whether poor outcomes are linked to acute kidney injury (AKI) developed during the course of the disease, or to pre-existing CKD ${ }^{39}$ a study conducted in Spain showed that patients with increased creatinine on admission, previous CKD or developing AKI had a higher risk of in-hospital death than those with normal creatinine on admission. ${ }^{40}$ Of note, the authors found that older age and diabetes, but not other comorbidities, were associated with in-hospital death. ${ }^{40}$ Finally, a study conducted in Mexico reported that patients with DM and CKD had a twofold higher rate of intubation, 56\% higher ICU admission and $21 \%$ excess probability of case fatality once admitted, than subjects with CKD alone. ${ }^{41}$ 
A. Subjects with diabetes

- In-hospital death

In-hospital death or IMV

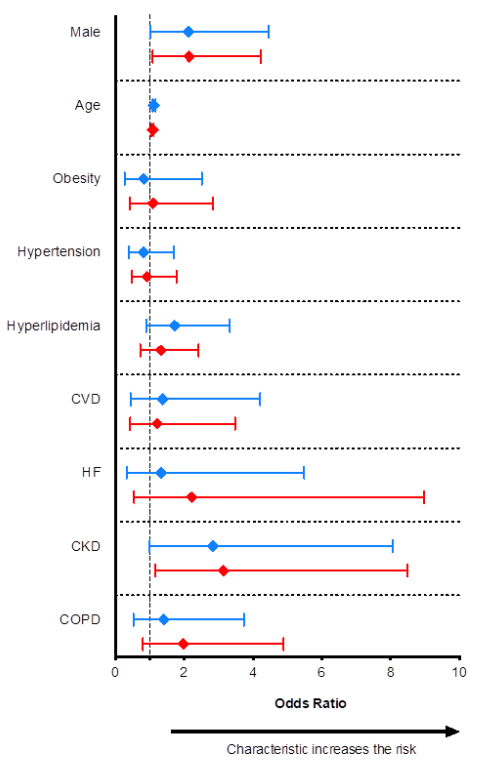

Odds ratio (95\% CI); P-value

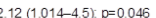
$2.14(1.1-4.2): p=0.029$

$1.12(1.06-1.2) ; p<0.001$ $1.08(1.05-1.1) ; p<0.001$

$2.84(1.1-8.1) ; p=0.05$ $3.14(1.2-8.5): p=0.024$

B. Subjects without diabetes In-hospital death or IMV

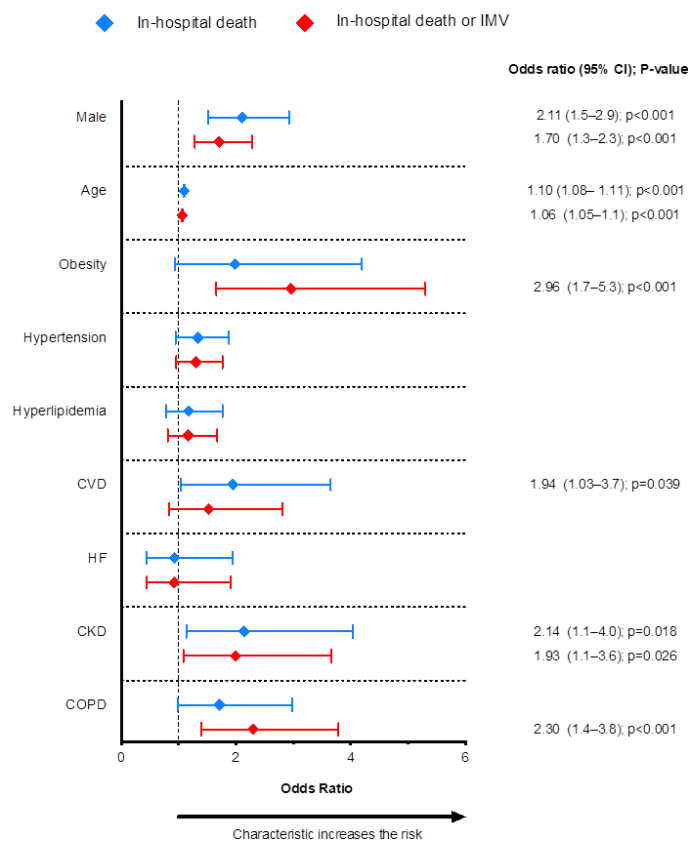

Figure 4 Clinical and demographic variables associated with increased risk of in-hospital death and the composite outcome of death and/or invasive mechanical ventilation (IMV) in subjects with diabetes (A) and without diabetes (B). CKD, chronic kidney disease; COPD, chronic obstructive pulmonary disease; CVD, cardiovascular disease; HF, heart failure.

In our study, we used splines as a scientific and preferable alternative to the categorisation of BG levels. ${ }^{42}$ We used this approach because a recent dose-response meta-analysis demonstrated a non-linear relationship between admission fasting blood glucose (FBG) level and COVID-19 severity, with high levels being significantly

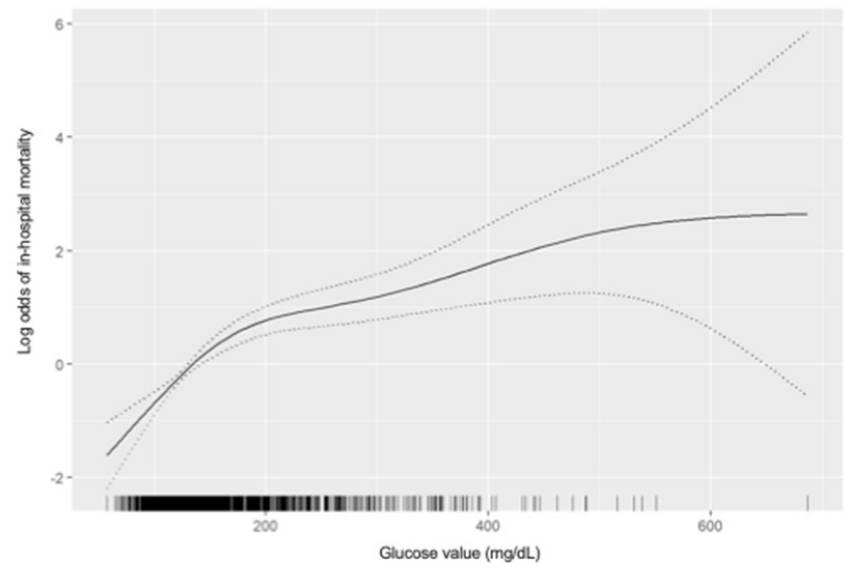

B

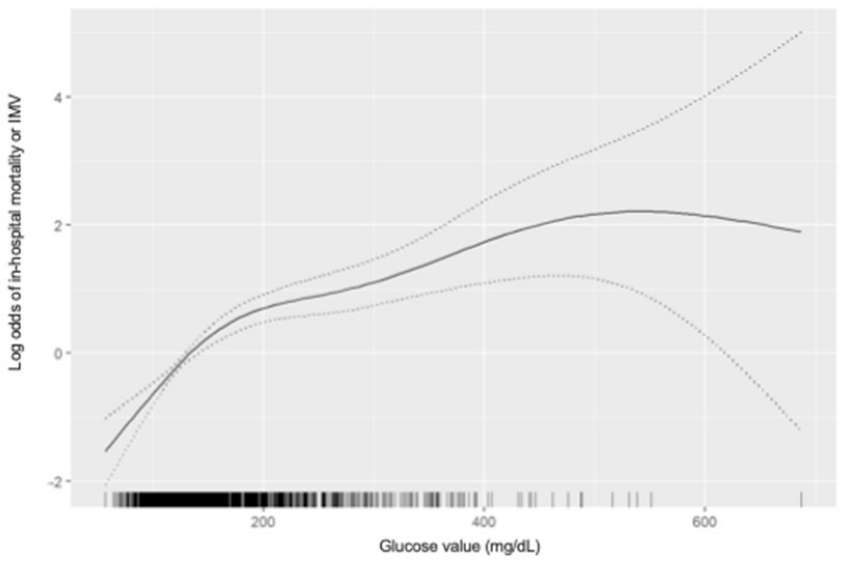

Figure 5 Spline plot demonstrating a marked non-linearity in the relationship between plasma glucose $(\mathrm{mg} / \mathrm{dL}$ ) levels on admission and the log odds of death (A) and death or invasive mechanical ventilation (IMV) rate (B). Tick marks above the horizontal axis indicate the values at which the observations were made. The dotted lines represent the $95 \% \mathrm{Cl}$. The model was adjusted for age, sex, obesity, hypertension, hyperlipidaemia, history of CVD, HF, CKD and COPD. CKD, chronic kidney disease; COPD, chronic obstructive pulmonary disease; CVD, cardiovascular disease; $\mathrm{HF}$, heart failure.

associated with increased mortality and poor outcome, regardless of pre-existing DM. ${ }^{43}$ These results confirmed previous observations that FBG on admission, and the odds of being admitted to the ICU, followed a logarithmic association, with different magnitudes of risk depending on the baseline level. ${ }^{42}$ We add to the literature that, besides the previously reported effect of hyperglycaemia on the risk of COVID-19 severity, ICU admission and mortality, ${ }^{24}{ }^{25}$ BG has a non-linear relationship with case fatality and the risk of death or IMV. It is possible that this relationship was also accompanied by, or reflected glycaemic variability and less time spent in range. Indeed, glycaemic fluctuation has been reported to be independently associated with poor prognosis and mortality in COVID-19 hospitalised patients. ${ }^{44}$ In the same vein, a study on ICU patients showed that the less time spent in 
A

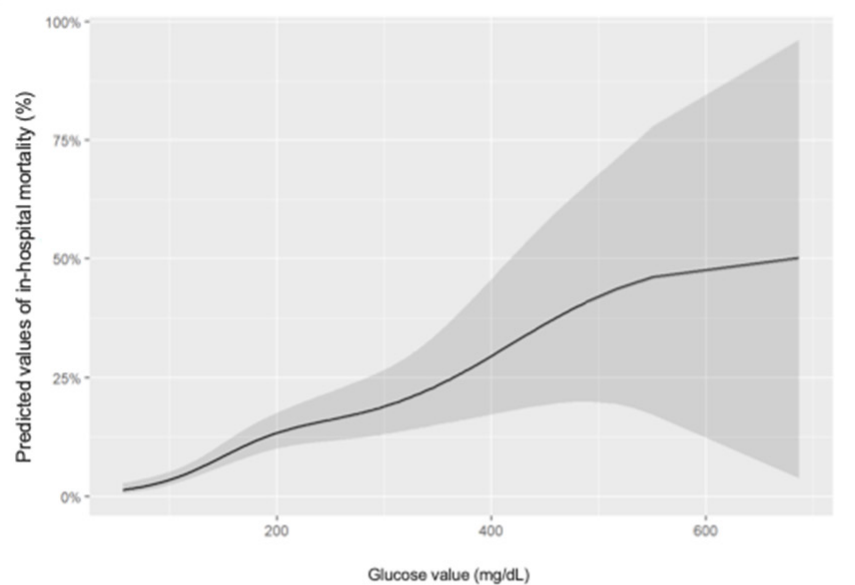

B

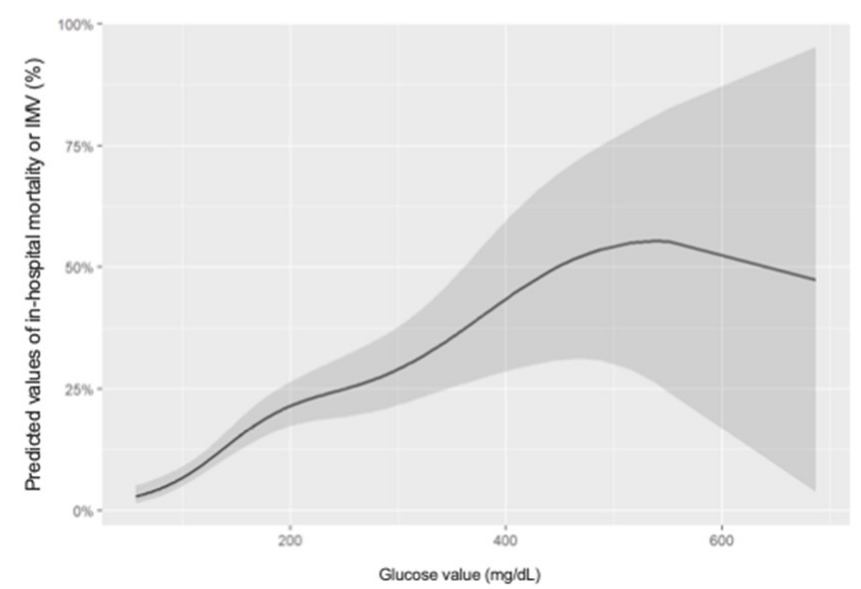

Figure 6 Predicted probability of in-hospital death $(A)$ and death or IMV (B) based on generalised smoothing splines. The shaded area represents the $95 \% \mathrm{Cl}$. The model was adjusted for age, sex, obesity, hypertension, hyperlipidaemia, history of CVD, HF, CKD and COPD. CKD, chronic kidney disease; COPD, chronic obstructive pulmonary disease; CVD, cardiovascular disease; HF, heart failure; IMV, invasive mechanical ventilation.

range was associated with increased utilisation of a ventilator, prolonged mechanical ventilation and increased mortality. ${ }^{45}$ Most importantly, a spline analysis of glucose levels in patients with DM with continuous glucose monitoring showed a non-linear relationship between time spent above range and glycaemic variability with the increased likelihood of composite adverse COVID-19 outcomes (need for ICU admission, mechanical ventilation or critical illness). ${ }^{46}$

\section{Limitations of this study}

The findings of this study must be interpreted with caution and a number of limitations should be borne in mind. First, we had limited data for SARS-CoV-2infected persons. For instance, we did not have access to the patient's medical history prior to admission; so the possibility exists that some important medical conditions were not included in the emergency room medical report and, therefore, not included in the analysis. Moreover, data on sociodemographic characteristics (ethnicity, race, economic or educational status) and toxic habits (smoking, alcohol or drug use) were not available. Second, we had very few registers for some important variables for diabetes, such as Hb1Ac (data from only 36 patients) and no data on weight or BMI. Indeed, no more than $10 \%$ of the patients had documented obesity, which is clearly lower than the expected prevalence in the general population. This was most probably related to the clinician's under-recording of this particular condition and to the fact that, during the first wave, obesity had not yet been identified as a significant risk factor and thus not specifically registered. Thirdly, the selection of DM subjects was made based on a proxy algorithm (including DM diagnosis during the hospital stay, antidiabetic treatment, and HbA1c and blood glucose levels at admission), which could have introduced selection or referral bias, potentially leading to an inaccurate estimation of DM prevalence. Besides, we had no access to the patients' treatments prior to hospital admission. Since the proportion of patients identified as having diabetes and receiving glucose-lowering agents was surprisingly low (approximately $40 \%$ ), this can be also attributed to antidiabetic treatment under-reporting at admission. Fourth, and inherent to data coming from hospital medical records, missing values could have reduced the statistical power of the study, or produced biased estimates. Fifth, we used random BG on admission for the spline analyses, thus preventing the distinction between stress-related hyperglycaemia and uncontrolled pre-existing DM. This also prevented the analysis of time in range or BG variability, both being linked to increased severity, case fatality and poor COVID-19 outcomes. ${ }^{42-46}$ Lastly, the study period coincides with the height of the first pandemic wave in Spain, when there was a shortage of ventilators and intensive care beds. At this point, age was the deciding factor for whether or not someone received potentially lifesaving ICU care. This might be reflected in our results, where in-hospital death was more frequent among those over 65 years, but ICU admission was more frequent among those $\leq 65$ years.

\section{CONCLUSIONS}

The results of our study confirm the high burden associated with DM in patients hospitalised due to SARS-CoV-2 infection. Comorbid DM poses a challenge to health professionals and the system because it is associated with severe disease, higher ICU admission rates, IMV and ultimately death, particularly among the elderly. The nonlinear relationship of hyperglycaemia at admission with increased odds of death and IMV suggests that optimising glycaemic control during the hospital stay could help to reduce in-hospital death and the composite death/IMV. Besides, out-of-hospital care should be a priority to reduce or prevent uncontrolled glycaemia among those with DM, as it could potentially help reduce poor outcomes when hospitalisation is required. 
Author affiliations

${ }^{1}$ DAP-Cat group, Unitat de Suport a la Recerca Barcelona, Fundació Institut Universitari per a la recerca a I'Atenció Primària de Salut Jordi Gol i Gurina (IDIAPJGol), Barcelona, Spain

${ }^{2}$ CIBER of Pathophysiology of Obesity and Nutrition (CIBEROBN), Instituto de Salud Carlos III (ISCIII), Madrid, Spain

${ }^{3}$ Department of Endocrinology and Nutrition, Institut d'Investigacions Biomèdiques August Pi i Sunyer, Hospital Clinic, Barcelona, Spain

${ }^{4}$ Department of Endocrinology and Nutrition, Hospital Universitari de la Santa Creu i Sant Pau, Barcelona, Spain

${ }^{5}$ Department of Medicine, Universitat Autònoma de Barcelona, Barcelona, Spain

${ }^{6} \mathrm{CIBER}$ of Bioengineering, Biomaterials and Nanomedicine (CIBER-BBN), Instituto de Salud Carlos III (ISCIII), Madrid, Spain

${ }^{7}$ Innovation office, Institut Català de la Salut, Barcelona, Spain

${ }^{8}$ Primary Health Care Center La Mina, Gerència d'Àmbit d'Atenció Primària Barcelona Ciutat, Institut Català de la Salut, Sant Adrià de Besòs, Spain ${ }^{9} \mathrm{CIBER}$ of Diabetes and Associated Metabolic Diseases (CIBERDEM), Instituto de Salud Carlos III (ISCIII), Madrid, Spain

${ }^{10}$ Infectious Diseases, Hospital Universitari de la Santa Creu i Sant Pau, Institut de Recerca Hospital Universitari de la Santa Creu i Sant Pau, Barcelona, Spain

${ }^{11}$ Diabetes Research Centre, University of Leicester, Leicester, UK

${ }^{12}$ Primary Health Care Center Raval Sud, Gerència d'Atenció Primaria, Institut Català de la Salut, Barcelona, Spain

${ }^{13}$ Departament of Medicine, University of Vic - Central University of Catalonia, Vic, Barcelona, Spain

\section{Twitter Emilio Ortega @EmilioOrtegaMdV and Didac Mauricio @DidacMauricio}

Acknowledgements Covid Data Save Lives-Hospitales HM for providing database. CIBER of Diabetes and Associated Metabolic Diseases (CIBERDEM) and CIBER of physiopathology of obesity and Nutrition (CIBEROBN) are initiatives from the Instituto de Salud Carlos III, Madrid, Spain. The authors acknowledge Amanda Prowse (Lochside Medical communications) for providing support in paper editing. KK is supported by the National Institute for Health Research (NIHR) Applied Research Collaboration East Midlands (ARC EM) and the NIHR Leicester Biomedical Research Centre (BRC).

Contributors Conceptualisation-E0, JF-N, RC, MM-C, BV, KK and DM. Methodology-EO, JF-N, RC, MM-C, BV, KK and DM. Formal analysis-RP-T and JR. Resources and data curation-RP-T, JR and BV. Writing (original draft preparation)—BV and MG. Writing (review and editing)—EO, JF-N, RC, MMC, MG, FXCC, EC, BV, KK, DM and PD. Supervision-DM, RC and JF-N. Project administration-BV.

Funding This study was supported by the Primary Care Diabetes Europe grant (grant number FEr20/0020).

Competing interests EO has received advisory and or speaking fees from AstraZeneca, Boehringer Ingelheim, Lilly, MSD, Novo Nordisk, Sanofi and Amgen; he received research grants to the institution from MSD and Amgen. RC has received advisory and/or speaking fees from Abbott, Ascensia, Lilly, MSD, Novo Nordisk and Sanofi. MM-C has received advisory honorarium from AstraZeneca, Bayer, Boehringer Ingelheim, GSK, Lilly, MSD, Novartis, Novo Nordisk and Sanofi; he received speaker honoraria from AstraZeneca, Bayer, Boehringer Ingelheim, GSK, Lilly, Menarini, MSD, Novartis, Novo Nordisk and Sanofi; he received research grants to the institution from AstraZeneca, GSK, Lilly, MSD, Novartis, Novo Nordisk and Sanofi. JF-N has received advisory and or speaking fees from AstraZeneca, Ascensia, Boehringer Ingelheim, GSK, Lilly, MSD, Novartis, Novo Nordisk and Sanofi; he received research grants to the institution from AstraZeneca, GSK, Lilly, MSD, Novartis, Novo Nordisk, Sanofi and Boehringer. KK has acted as a consultant, speaker or received grants for investigator-initiated studies for AstraZeneca, Novartis, Novo Nordisk, Sanofi-Aventis, Lilly and Merck Sharp \& Dohme, Boehringe Ingelheim, Bayer, Berlin-Chemie AG/Menarini Group, Janssen and Napp. DM has received advisory and/or speaking fees from AstraZeneca, Ascensia, Boehringer Ingelheim, GSK, Lilly, MSD, Novartis, Novo Nordisk and Sanofi; he received research grants to the institution from AstraZeneca, GSK, Lilly, MSD, Novartis, Novo Nordisk, Sanofi and Boehringer. PD has received lecture and Advisory Board fees from Gilead Sciences, Roche, Merck, Sharp \& Dohme, ViiV Healthcare, Janssen \& Cilag, Theratechnologies, Boehringer Ingelheim and Ferrer International. PD has received research grants from Gilead Sciences, ViiV Healthcare, GSK, Janssen \& Cilag and Boehringer Ingelheim. BV, FXCC, JR, RP-T, MG and EC have no conflict of interest to declare.

Patient consent for publication Not required.
Ethics approval The study was approved by the Ethics Committee of the Primary Health Care University Research Institute (IDIAP) Jordi Gol, Barcelona (approval number: 20/089-PCV). This study does not involve animal subjects.

Provenance and peer review Not commissioned; externally peer reviewed.

Data availability statement Data may be obtained from a third party and are not publicly available.

Supplemental material This content has been supplied by the author(s). It has not been vetted by BMJ Publishing Group Limited (BMJ) and may not have been peer-reviewed. Any opinions or recommendations discussed are solely those of the author(s) and are not endorsed by BMJ. BMJ disclaims all liability and responsibility arising from any reliance placed on the content. Where the content includes any translated material, BMJ does not warrant the accuracy and reliability of the translations (including but not limited to local regulations, clinical guidelines, terminology, drug names and drug dosages), and is not responsible for any error and/or omissions arising from translation and adaptation or otherwise.

Open access This is an open access article distributed in accordance with the Creative Commons Attribution Non Commercial (CC BY-NC 4.0) license, which permits others to distribute, remix, adapt, build upon this work non-commercially, and license their derivative works on different terms, provided the original work is properly cited, appropriate credit is given, any changes made indicated, and the use is non-commercial. See: http://creativecommons.org/licenses/by-nc/4.0/.

\section{ORCID iDs}

Manel Mata-Cases http://orcid.org/0000-0003-3693-3622

Esmeralda Castelblanco http://orcid.org/0000-0002-2061-6270

Didac Mauricio http://orcid.org/0000-0002-2868-0250

\section{REFERENCES}

1 World Health Organization. 15-Novel Coronavirus(2019-nCoV). WHO Bull 2020:1-7.

2 Agència de Salut Pública de Catalunya (ASPCAT). Informe tècnic resum dels casos de covid-19 a Catalunya, 2020. Available: http:// salutpublica.gencat.cat/web/.content/minisite/aspcat/butlletins/ vigilanciaaspcat/2020/45/INFORME-TECNIC-3-COVID-19_020420. pdf

3 Linde P. Sanidad confirma en La Gomera El primer caso de coronavirus en España. El País. ISSN 1134-6582, February 1, 2020. Available: https://elpais.com/sociedad/2020/01/31/actualidad/ 1580509404_469734.htm

4 Muniyappa R, Gubbi S. COVID-19 pandemic, coronaviruses, and diabetes mellitus. Am J Physiol Endocrinol Metab 2020;318:E736-41.

5 Zhou Y, Yang Q, Chi J, et al. Comorbidities and the risk of severe or fatal outcomes associated with coronavirus disease 2019: a systematic review and meta-analysis. Int $J$ Infect Dis 2020;99:47-56.

6 Deng G, Yin M, Chen X, et al. Clinical determinants for fatality of 44,672 patients with COVID-19. Crit Care 2020;24:179.

7 Mesas AE, Cavero-Redondo I, Álvarez-Bueno C, et al. Predictors of in-hospital COVID-19 mortality: a comprehensive systematic review and meta-analysis exploring differences by age, sex and health conditions. PLoS One 2020;15:e0241742.

8 Pendu JL, Breiman A, Rocher J, et al. Abo blood types and COVID-19: spurious, anecdotal, or truly important relationships? A Reasoned review of available data. Viruses 2021;13:160.

9 Sardu C, Marfella R, Maggi P, et al. Implications of AB0 blood group in hypertensive patients with covid-19. BMC Cardiovasc Disord 2020;20:373.

10 Hoffmann M, Kleine-Weber H, Schroeder S, et al. SARS-CoV-2 cell entry depends on ACE2 and TMPRSS2 and is blocked by a clinically proven protease inhibitor. Cell 2020;181:271-80.

11 Baral R, Tsampasian V, Debski M, et al. Association between reninangiotensin-aldosterone system inhibitors and clinical outcomes in patients with COVID-19: a systematic review and meta-analysis. JAMA Netw Open 2021;4:e213594.

12 Matarese A, Gambardella J, Sardu C, et al. miR-98 regulates TMPRSS2 expression in human endothelial cells: key implications for COVID-19. Biomedicines 2020;8:462.

13 Papazafiropoulou AK, Antonopoulos S. The COVID-19 pandemic and diabetes mellitus. Archives of medical sciences. Atherosclerotic Diseases 2020:5: 200 .

14 McDonald HI, Nitsch D, Millett ERC, et al. New estimates of the burden of acute community-acquired infections among older people with diabetes mellitus: a retrospective cohort study using linked electronic health records. Diabet Med 2014;31:606-14. 
15 Li S, Wang J, Zhang B, et al. Diabetes mellitus and causespecific mortality: a population-based study. Diabetes Metab J 2019;43:319-41.

16 Del Sole F, Farcomeni A, Loffredo L, et al. Menichelli D, Vicario T, et al. Features of severe COVID-19: A systematic review and metaanalysis. Eur J Clin Invest 2020;50:0-1.

17 Pugliese G, Vitale M, Resi V, et al. Is diabetes mellitus a risk factor for coronavirus disease 19 (COVID-19)? Acta Diabetol 2020;19.

18 Peric S, Stulnig TM. Diabetes and COVID-19. Wien Klin Wochenschr 2020;132:356-61.

19 Barron E, Bakhai C, Kar P, et al. Associations of type 1 and type 2 diabetes with COVID-19-related mortality in England: a wholepopulation study. Lancet Diabetes Endocrinol 2020;8:813-822.

20 Tian W, Jiang W, Yao J, et al. Predictors of mortality in hospitalized COVID-19 patients: a systematic review and meta-analysis. J Med Virol 2020

21 Mantovani A, Byrne CD, Zheng $\mathrm{M}-\mathrm{H}$, et al. Diabetes as a risk factor for greater COVID-19 severity and in-hospital death: a meta-analysis of observational studies. Nutr Metab Cardiovasc Dis 2020;30:1236-48.

22 Wu C, Chen X, Cai Y, et al. Risk factors associated with acute respiratory distress syndrome and death in patients with coronavirus disease 2019 pneumonia in Wuhan, China. JAMA Intern Med 2020;180:934-43.

$23 \mathrm{Li} \mathrm{X}, \mathrm{Xu} \mathrm{S}, \mathrm{Yu} \mathrm{M}$, et al. Risk factors for severity and mortality in adult COVID-19 inpatients in Wuhan. J Allergy Clin Immunol 2020;146:110-8

24 Lee $\mathrm{MH}$, Wong $\mathrm{C}, \mathrm{Ng} \mathrm{CH}$, et al. Effects of hyperglycaemia on complications of COVID-19: a meta-analysis of observational studies. Diabetes Obes Metab 2021;23:287-9.

25 Yang Y, Cai Z, Zhang J. Hyperglycemia at admission is a strong predictor of mortality and severe/critical complications in COVID-19 patients: a meta-analysis. Biosci Rep 2021;41. doi:10.1042/ BSR20203584. [Epub ahead of print: 2602 2021].

26 Marfella R, Paolisso P, Sardu C, et al. Negative impact of hyperglycaemia on tocilizumab therapy in Covid-19 patients. Diabetes Metab 2020;46:403-5.

27 Soriguer F, Goday A, Bosch-Comas A, et al. Prevalence of diabetes mellitus and impaired glucose regulation in Spain: the Di@bet.es Study. Diabetologia 2012;55:88-93.

28 Caballero AE, Ceriello A, Misra A, et al. COVID-19 in people living with diabetes: an international consensus. J Diabetes Complications 2020;34:107671.

29 Wood S. mgcv: mixed GAM computation vehicle with automatic smoothness estimation. R-package version 1.8-31, 2019. Available: https://CRAN.R-project.org/package=mgcv

30 Li G, Deng Q, Feng J, et al. Clinical characteristics of diabetic patients with COVID-19. J Diabetes Res 2020;2020:1652403.

31 Izcovich A, Ragusa MA, Tortosa F, et al. Prognostic factors for severity and mortality in patients infected with COVID-19: a systematic review. PLoS One 2020;15:e0241955.
32 Cariou B, Hadjadj S, Wargny M, et al. Phenotypic characteristics and prognosis of inpatients with COVID-19 and diabetes: the CORONADO study. Diabetologia 2020;63:1500-15.

33 Zhu L, She Z-G, Cheng X, et al. Association of blood glucose control and outcomes in patients with COVID-19 and pre-existing type 2 diabetes. Cell Metab 2020;31:1068-77.

34 Elamari S, Motaib I, Zbiri S, et al. Characteristics and outcomes of diabetic patients infected by the SARS-CoV-2. Pan Afr Med J 2020;37:32. Epub ahead of print.

35 Yan Y, Yang Y, Wang F, et al. Clinical characteristics and outcomes of patients with severe covid-19 with diabetes. BMJ Open Diabetes Res Care 2020;8:e001343.

36 Laguna-Goya R, Utrero-Rico A, Talayero P, et al. IL-6-based mortality risk model for hospitalized patients with COVID-19. J Allergy Clin Immunol 2020;146:799-807.

37 Working group for the surveillance and control of COVID-19 in Spain, Members of the Working group for the surveillance and control of COVID-19 in SpainRedondo-Bravo L. The first wave of the COVID-19 pandemic in Spain: characterisation of cases and risk factors for severe outcomes, as at 27 April 2020. Euro Surveill 2020;25:2001431.

38 Tian W, Jiang W, Yao J, et al. Predictors of mortality in hospitalized COVID-19 patients: a systematic review and meta-analysis. J Med Virol 2020;92:1875-83.

39 Carlson N, Nelveg-Kristensen K-E, Freese Ballegaard E, et al. Increased vulnerability to COVID-19 in chronic kidney disease. $J$ Intern Med 2021;290:166-78.

40 Portolés J, Marques M, López-Sánchez P, et al. Chronic kidney disease and acute kidney injury in the COVID-19 Spanish outbreak. Nephrol Dial Transplant 2020;35:1353-61.

41 Leon-Abarca JA, Memon RS, Rehan B, et al. The impact of COVID-19 in diabetic kidney disease and chronic kidney disease: a population-based study. Acta Biomed 2020;91:e2020161.

42 Alahmad B, Al-Shammari AA, Bennakhi A, et al. Fasting blood glucose and COVID-19 severity: nonlinearity matters. Diabetes Care 2020;43:3113-6.

43 Lazarus G, Audrey J, Wangsaputra VK, et al. High admission blood glucose independently predicts poor prognosis in COVID-19 patients: a systematic review and dose-response meta-analysis. Diabetes Res Clin Pract 2021;171:108561.

44 Chen L, Sun W, Liu Y, et al. Association of early-phase in-hospital glycemic fluctuation with mortality in adult patients with coronavirus disease 2019. Diabetes Care 2021;44:dc200780.

45 Kapoor R, Timsina LR, Gupta N. Maintaining blood glucose levels in range (70-150 mg/dL) is difficult in COVID-19 compared to Non-COVID-19 ICU Patients - A retrospective analysis. J Clin Med 2020;9:3635

46 Shen Y, Fan X, Zhang L, et al. Thresholds of glycemia and the outcomes of COVID-19 complicated with diabetes: a retrospective exploratory study using continuous glucose monitoring. Diabetes Care 2021;44:dc201448. 